\title{
How Does a Microbial Rhodopsin RxR Realize Its Exceptionally High Thermostability with the Proton-Pumping Function Being Retained?
}

Tomohiko Hayashi, ${ }^{1, \#}$ Satoshi Yasuda, ${ }^{1,2,3}$ Kano Suzuki, ${ }^{2}$ Tomoki Akiyama, ${ }^{2}$ Kanae Kanehara, ${ }^{4}$ Keiichi Kojima, ${ }^{4,5}$ Mikio Tanabe, ${ }^{6}$ Ryuichi Kato, ${ }^{6}$ Toshiya Senda, ${ }^{6}$ Yuki Sudo, ${ }^{4,5}$ Takeshi Murata, ${ }^{*, 2,3}$ and Masahiro Kinoshita**,1

${ }^{1}$ Institute of Advanced Energy, Kyoto University, Gokasho, Uji, Kyoto 611-0011, Japan,

${ }^{2}$ Graduate School of Science, Chiba University, 1-33 Yayoi-cho, Inage, Chiba 263-8522, Japan,

${ }^{3}$ Molecular Chirality Research Center, Chiba University, 1-33 Yayoi-cho, Inage, Chiba 263-8522, Japan,

${ }^{4}$ Division of Pharmaceutical Sciences, Okayama University, 1-1 Tsushimanaka, Kita, Okayama, Okayama 700-8530, Japan,

${ }^{5}$ Graduate School of Medicine, Dentistry and Pharmaceutical Sciences, Okayama University, Okayama 700-8530, Japan

${ }^{6}$ Structural Biology Research Center, Institute of Materials Structure Science, High Energy Accelerator Research Organization (KEK), 1-1 Oho, Tsukuba, Ibaraki 305-0801, Japan

\#Present address: Interdisciplinary Program of Biomedical Engineering, Assistive Technology, and Art and Sports Sciences, Faculty of Engineering, Niigata University, 8050 Ikarashi 2-no-cho, Nishi-ku, Niigata, 950-2181, Japan

\footnotetext{
* To whom correspondence should be addressed. Tel: +81-43-290-2794. E-mail: t.murata@faculty.chiba-u.jp.

** To whom correspondence should be addressed. Tel: +81-774-38-3503. E-mail: kinoshit@iae.kyoto-u.ac.jp.
} 


\section{Effects of the Values of $r_{c}$ and $N_{c}$ Set in Molecular Dynamics Simulation}

The result presented in the main text is for the setting, $r_{\mathrm{C}}=5 \AA$ and $N_{\mathrm{C}}=2.5$. We emphasize that only the energetic component is influenced by the setting. Our conclusions are robust against a change of $N_{\mathrm{C}}$ to 2.0 or 3.0 and that of $r_{\mathrm{C}}$ to $6 \AA$, as corroborated in Tables S1 through S5 presented below. The number of residues in each of the TM, EC, and IC portions determined is as follows: $N_{\mathrm{r}, \mathrm{TM}}=138, N_{\mathrm{r}, \mathrm{EC}}=36$, and $N_{\mathrm{r}, \mathrm{IC}}=55$ for RxR; $N_{\mathrm{r}, \mathrm{TM}}=136, N_{\mathrm{r}, \mathrm{EC}}=42$, and $N_{\mathrm{r}, \mathrm{IC}}=48$ for HsBR. $N_{\mathrm{r}}=229$ for RxR and $N_{\mathrm{r}}=226$ for HsBR $\left(N_{\mathrm{r}}=N_{\mathrm{r}, \mathrm{TM}}+N_{\mathrm{r}, \mathrm{EC}}+N_{\mathrm{r}, \mathrm{IC}}\right)$.

RxR is more stable than HsBR in terms of $-\Delta S_{\mathrm{VH}} /\left(k_{\mathrm{B}} N_{\mathrm{r}}\right)$ by -0.27 in Tables S1-S5. RxR is more stable than HsBR in terms of $\Delta \Lambda /\left(k_{\mathrm{B}} T_{0} N_{\mathrm{r}}\right)$ by $-0.26,-0.01,-0.26,-0.32$, and -0.28 in Tables S1-S5, respectively. Irrespective of the values of $r_{\mathrm{C}}$ and $N_{\mathrm{C}}$ set, $\mathrm{RxR}$ is predicted to be more stable both entropically and energetically.

Table S1. $\Delta F /\left(k_{\mathrm{B}} T_{0} N_{\mathrm{r}}\right)$ and Its Entropic $\left(-\Delta S_{\mathrm{vH}} /\left(k_{\mathrm{B}} N_{\mathrm{r}}\right)\right)$ and Energetic $\left(\Delta /\left(k_{\mathrm{B}} T_{0} N_{\mathrm{r}}\right)\right)$ Components $\left(r_{\mathrm{C}=5} \AA \text { and } N_{\mathrm{C}=2.0}\right)^{a}$

\begin{tabular}{|c|c|c|c|c|c|c|}
\hline & $\begin{array}{c}-\Delta S \mathrm{VH} / k_{\mathrm{B}} \\
\left(N_{\mathrm{r}, \text { helices }}\right)\end{array}$ & $\begin{array}{c}-\Delta S \mathrm{VH}_{\mathrm{H}} /\left(k_{\mathrm{B}} N_{\mathrm{r}}\right) \\
\left(N_{\mathrm{r}}\right)\end{array}$ & $\Delta \Lambda_{\mathrm{TM}} /\left(k_{\mathrm{B}} T_{0}\right)$ & $\Delta \Lambda_{\mathrm{WI}} /\left(k_{\mathrm{B}} T_{0}\right)$ & $\begin{array}{c}\Delta M /\left(k_{\mathrm{B}} T_{0} N_{\mathrm{r}}\right) \\
\left(N_{\mathrm{r}}\right)\end{array}$ & $\Delta F /\left(k_{\mathrm{B}} T_{0} N_{\mathrm{r}}\right)$ \\
\hline $\mathrm{RxR}$ & $\begin{array}{c}-669.4 \\
(185)\end{array}$ & $\begin{array}{l}-2.92 \\
(229)\end{array}$ & -535.7 & 84.0 & $\begin{array}{l}-1.97 \\
(229)\end{array}$ & -4.90 \\
\hline HsBR & $\begin{array}{c}-599.9 \\
(170)\end{array}$ & $\begin{array}{l}-2.65 \\
(226)\end{array}$ & -511.6 & 126.0 & $\begin{array}{l}-1.71 \\
(226)\end{array}$ & -4.36 \\
\hline
\end{tabular}

${ }^{a} \Delta \Lambda_{\mathrm{TM}} /\left(k_{\mathrm{B}} T_{0}\right)$ and $\Delta \Lambda_{\mathrm{WI}} /\left(k_{\mathrm{B}} T_{0}\right)$ are the energetic components for the TM and WI regions, respectively: $\Delta \Lambda /\left(k_{\mathrm{B}} T_{0}\right)=\Delta \Lambda_{\mathrm{TM}} /\left(k_{\mathrm{B}} T_{0}\right)+\Delta \Lambda_{\mathrm{wI}} /\left(k_{\mathrm{B}} T_{0}\right) . N_{\mathrm{r} \text {,helices }}$ is the number of residues in the seven helices. 
Table S2. $\Delta F /\left(k_{\mathrm{B}} T_{0} N_{\mathrm{r}}\right)$ and Its Entropic $\left(-\Delta S_{\mathrm{VH}} /\left(k_{\mathrm{B}} N_{\mathrm{r}}\right)\right)$ and Energetic $\left(\Delta / /\left(k_{\mathrm{B}} T_{0} N_{\mathrm{r}}\right)\right)$ Components $\left(r_{\mathrm{C}=5} \AA \text { and } N_{\mathrm{C}}=3.0\right)^{a}$

\begin{tabular}{|c|c|c|c|c|c|c|}
\hline & $\begin{array}{c}-\Delta S_{\mathrm{VH}} / k_{\mathrm{B}} \\
\left(N_{\mathrm{r}, \mathrm{helices}}\right)\end{array}$ & $\begin{array}{c}-\Delta S_{\mathrm{VH}} /\left(k_{\mathrm{B}} N_{\mathrm{r}}\right) \\
\left(N_{\mathrm{r}}\right)\end{array}$ & $\Delta \Lambda_{\mathrm{TM}} /\left(k_{\mathrm{B}} T_{0}\right)$ & $\Delta \Lambda_{\mathrm{w}} /\left(k_{\mathrm{B}} T_{0}\right)$ & $\begin{array}{c}\Delta M /\left(k_{\mathrm{B}} T_{0} N_{\mathrm{r}}\right) \\
\left(N_{\mathrm{r}}\right)\end{array}$ & $\Delta F /\left(k_{\mathrm{B}} T_{0} N_{\mathrm{r}}\right)$ \\
\hline $\mathrm{RxR}$ & $\begin{array}{c}-669.4 \\
(185)\end{array}$ & $\begin{array}{l}-2.92 \\
(229)\end{array}$ & -583.1 & 84.0 & $\begin{array}{l}-2.18 \\
(229)\end{array}$ & -5.10 \\
\hline HsBR & $\begin{array}{c}-599.9 \\
(170)\end{array}$ & $\begin{array}{l}-2.65 \\
(226)\end{array}$ & -596.0 & 105.0 & $\begin{array}{l}-2.17 \\
(226)\end{array}$ & -4.83 \\
\hline
\end{tabular}

${ }^{a} \Delta \Lambda_{\mathrm{TM}} /\left(k_{\mathrm{B}} T_{0}\right)$ and $\Delta \Lambda_{\mathrm{WI}} /\left(k_{\mathrm{B}} T_{0}\right)$ are the energetic components for the TM and WI regions, respectively: $\Delta \Lambda /\left(k_{\mathrm{B}} T_{0}\right)=\Delta \Lambda_{\mathrm{TM}} /\left(k_{\mathrm{B}} T_{0}\right)+\Delta \Lambda_{\mathrm{WI}} /\left(k_{\mathrm{B}} T_{0}\right) . N_{\mathrm{r} \text {,helices }}$ is the number of residues in the seven helices.

Table S3. $\Delta F /\left(k_{\mathrm{B}} T_{0} N_{\mathrm{r}}\right)$ and Its Entropic $\left(-\Delta S_{\mathrm{VH}} /\left(k_{\mathrm{B}} N_{\mathrm{r}}\right)\right)$ and Energetic $\left(\Delta \Lambda /\left(k_{\mathrm{B}} T_{0} N_{\mathrm{r}}\right)\right)$ Components $\left(r \mathrm{C}=6 \AA \text { and } N_{\mathrm{C}}=2.5\right)^{a}$

\begin{tabular}{|c|c|c|c|c|c|c|}
\hline & $\begin{array}{c}-\Delta S_{\mathrm{VH}} / k_{\mathrm{B}} \\
\left(N_{\mathrm{r}, \text { helices }}\right)\end{array}$ & $\begin{array}{c}-\Delta S_{\mathrm{VH}} /\left(k_{\mathrm{B}} N_{\mathrm{r}}\right) \\
\left(N_{\mathrm{r}}\right)\end{array}$ & $\Delta \Lambda_{\mathrm{TM}} /\left(k_{\mathrm{B}} T_{0}\right)$ & $\Delta M_{\mathrm{WI}} /\left(k_{\mathrm{B}} T_{0}\right)$ & $\begin{array}{c}\Delta M /\left(k_{\mathrm{B}} T_{0} N_{\mathrm{r}}\right) \\
\left(N_{\mathrm{r}}\right)\end{array}$ & $\Delta F /\left(k_{\mathrm{B}} T_{0} N_{\mathrm{r}}\right)$ \\
\hline $\mathrm{RxR}$ & $\begin{array}{c}-669.4 \\
(185)\end{array}$ & $\begin{array}{l}-2.92 \\
(229)\end{array}$ & -500.0 & 84.0 & $\begin{array}{l}-1.82 \\
(229)\end{array}$ & -4.74 \\
\hline HsBR & $\begin{array}{c}-599.9 \\
(170)\end{array}$ & $\begin{array}{l}-2.65 \\
(226)\end{array}$ & -485.5 & 133.0 & $\begin{array}{l}-1.56 \\
(226)\end{array}$ & -4.22 \\
\hline
\end{tabular}

${ }^{a} \Delta \Lambda_{\mathrm{TM}} /\left(k_{\mathrm{B}} T_{0}\right)$ and $\Delta \Lambda_{\mathrm{WI}} /\left(k_{\mathrm{B}} T_{0}\right)$ are the energetic components for the TM and WI regions, respectively: $\Delta \Lambda /\left(k_{\mathrm{B}} T_{0}\right)=\Delta \Lambda_{\mathrm{TM}} /\left(k_{\mathrm{B}} T_{0}\right)+\Delta \Lambda_{\mathrm{W}} /\left(k_{\mathrm{B}} T_{0}\right) . N_{\mathrm{r} \text {,helices }}$ is the number of residues in the seven helices. 
Table S4. $\Delta F /\left(k_{\mathrm{B}} T_{0} N_{\mathrm{r}}\right)$ and Its Entropic $\left(-\Delta S_{\mathrm{VH}} /\left(k_{\mathrm{B}} N_{\mathrm{r}}\right)\right)$ and Energetic $\left(\Delta / /\left(k_{\mathrm{B}} T_{0} N_{\mathrm{r}}\right)\right)$ Components $\left(r_{\mathrm{C}=6} \AA \text { and } N_{\mathrm{C}}=2.0\right)^{a}$

\begin{tabular}{|c|c|c|c|c|c|c|}
\hline & $\begin{array}{c}-\Delta S_{\mathrm{VH}} / k_{\mathrm{B}} \\
\left(N_{\mathrm{r}, \text { helices }}\right)\end{array}$ & $\begin{array}{c}-\Delta S_{\mathrm{VH}} /\left(k_{\mathrm{B}} N_{\mathrm{r}}\right) \\
\left(N_{\mathrm{r}}\right)\end{array}$ & $\Delta \Lambda_{\mathrm{TM}} /\left(k_{\mathrm{B}} T_{0}\right)$ & $\Delta \Lambda_{\mathrm{wI}} /\left(k_{\mathrm{B}} T_{0}\right)$ & $\begin{array}{c}\Delta \Lambda /\left(k_{\mathrm{B}} T_{0} N_{\mathrm{r}}\right) \\
\left(N_{\mathrm{r}}\right)\end{array}$ & $\Delta F /\left(k_{\mathrm{B}} T_{0} N_{\mathrm{r}}\right)$ \\
\hline $\mathrm{RxR}$ & $\begin{array}{c}-669.4 \\
(185)\end{array}$ & $\begin{array}{l}-2.92 \\
(229)\end{array}$ & -496.7 & 91.0 & $\begin{array}{l}-1.77 \\
(229)\end{array}$ & -4.69 \\
\hline HsBR & $\begin{array}{c}-599.9 \\
(170)\end{array}$ & $\begin{array}{l}-2.65 \\
(226)\end{array}$ & -467.3 & 140.0 & $\begin{array}{l}-1.45 \\
(226)\end{array}$ & -4.10 \\
\hline
\end{tabular}

${ }^{a} \Delta \Lambda_{\mathrm{TM}} /\left(k_{\mathrm{B}} T_{0}\right)$ and $\Delta \Lambda_{\mathrm{WI}} /\left(k_{\mathrm{B}} T_{0}\right)$ are the energetic components for the TM and WI regions, respectively: $\Delta \Lambda /\left(k_{\mathrm{B}} T_{0}\right)=\Delta \Lambda_{\mathrm{TM}} /\left(k_{\mathrm{B}} T_{0}\right)+\Delta \Lambda_{\mathrm{WI}} /\left(k_{\mathrm{B}} T_{0}\right) . N_{\mathrm{r} \text {,helices }}$ is the number of residues in the seven helices.

Table S5. $\Delta F /\left(k_{\mathrm{B}} T_{0} N_{\mathrm{r}}\right)$ and Its Entropic $\left(-\Delta S_{\mathrm{vH}_{\mathrm{H}}} /\left(k_{\mathrm{B}} N_{\mathrm{r}}\right)\right)$ and Energetic $\left(\Delta / /\left(k_{\mathrm{B}} T_{0} N_{\mathrm{r}}\right)\right)$ Components $\left(r_{\mathrm{C}=6} \AA \text { and } N_{\mathrm{C}}=3.0\right)^{a}$

\begin{tabular}{|c|c|c|c|c|c|c|}
\hline & $\begin{array}{c}-\Delta S_{\mathrm{VH}} / k_{\mathrm{B}} \\
\left(N_{\mathrm{r}, \text { helices }}\right)\end{array}$ & $\begin{array}{c}-\Delta S_{\mathrm{VH}} /\left(k_{\mathrm{B}} N_{\mathrm{r}}\right) \\
\left(N_{\mathrm{r}}\right)\end{array}$ & $\Delta \Lambda_{\mathrm{TM}} /\left(k_{\mathrm{B}} T_{0}\right)$ & $\Delta \Lambda_{\mathrm{WI}} /\left(k_{\mathrm{B}} T_{0}\right)$ & $\begin{array}{c}\Delta M /\left(k_{\mathrm{B}} T_{0} N_{\mathrm{r}}\right) \\
\left(N_{\mathrm{r}}\right)\end{array}$ & $\Delta F /\left(k_{\mathrm{B}} T_{0} N_{\mathrm{r}}\right)$ \\
\hline $\mathrm{RxR}$ & $\begin{array}{c}-669.4 \\
(185)\end{array}$ & $\begin{array}{l}-2.92 \\
(229)\end{array}$ & -519.9 & 84.0 & $\begin{array}{l}-1.90 \\
(229)\end{array}$ & -4.83 \\
\hline HsBR & $\begin{array}{c}-599.9 \\
(170)\end{array}$ & $\begin{array}{l}-2.65 \\
(226)\end{array}$ & -492.5 & 126.0 & $\begin{array}{l}-1.62 \\
(226)\end{array}$ & -4.28 \\
\hline
\end{tabular}

${ }^{a} \Delta \Lambda_{\mathrm{TM}} /\left(k_{\mathrm{B}} T_{0}\right)$ and $\Delta \Lambda_{\mathrm{WI}} /\left(k_{\mathrm{B}} T_{0}\right)$ are the energetic components for the TM and WI regions, respectively: $\Delta M /\left(k_{\mathrm{B}} T_{0}\right)=\Delta \Lambda_{\mathrm{TM}} /\left(k_{\mathrm{B}} T_{0}\right)+\Delta \Lambda_{\mathrm{WI}} /\left(k_{\mathrm{B}} T_{0}\right) . N_{\mathrm{r} \text {,helices }}$ is the number of residues in the seven helices. 